\section{Urinoma gigante postraumático asintomático}

Calvo-Vázquez I, Hernández-Méndez EA, Cortés-Raygoza P, Ortega-González $\mathrm{ME}$, Sánchez-Aquino U, Veliz-Cabrera G y colaboradores.

\section{Resumen}

ANTECEDENTES: el urinoma es una colección retroperitoneal de orina espontánea o secundaria a traumatismos, que es resultado de una obstrucción o pérdida de la continuidad de la vía urinaria, con función renal conservada.

CASO CLÍNICO: paciente masculino de 23 años de edad, con antecedente de fractura expuesta de radio y cúbito derecho, provocada por un accidente vehicular. Dos meses después del traumatismo inició con aumento de volumen en la región lumbar y el flanco derecho. La urotomografía reportó una colección con densidad líquida (22 UH), desde el diafragma hasta la región sacroiliaca derecha, que concentró el medio de contraste. La pielografía ascendente derecha evidenció la fuga extensa del medio de contraste del uréter derecho, en el tercio superior. En la exploración quirúrgica se drenaron 7 litros de orina, además de observar la disección del músculo oblicuo y transverso, comunicación con el retroperitoneo, avulsión del uréter derecho (en el tercio superior) y tejido necrótico e inflamatorio. Se efectuó la nefrectomía subcapsular, cuyo reporte de patología fue: hemorragia multifocal reciente, cálices y pelvis renal seccionados, con bordes de la pelvis renal necróticos e infiltrado inflamatorio. Tres semanas después del posoperatorio se realizó una tomografía, con hallazgo de colección en el sitio quirúrgico, tratada con drenaje percutáneo sin complicaciones ni incidencias adicionales.

CONCLUSIONES: el retraso en el diagnóstico de los urinomas se debe a la aparición gradual de los síntomas, pues sólo existe dolor lumbar en caso de infección. El tratamiento de elección consiste en drenaje percutáneo o nefrectomía.

PALABRAS CLAVE: urinoma, urinoma postraumático.
Hospital General Dr. Manuel Gea González (SSA), Ciudad de México.

Recibido: septiembre 2017

Aceptado: octubre 2017

Correspondencia

Dr. Ivan Calvo Vázquez

dr.ivancv_unam@hotmail.com

Este artículo debe citarse como

Calvo-Vázquez I, Hernández-Méndez EA, CortésRaygoza P, Ortega-González ME, Sánchez-Aquino $U$, Veliz-Cabrera G, y col. Urinoma gigante postraumático asintomático. Rev Mex Urol. 2017 novdic;77(6):464-469.

DOI: https://doi.org/10.24245/revmexurol.v77i6.1633 
CLINICAL CASE: A 23-year-old man had a past medical history of an exposed fracture of the right radius and ulna from a vehicular accident. Two months after the trauma, he noticed an increase in volume in the lumbar region and right flank. A computed tomography urogram showed a collection with liquid density ( $22 \mathrm{HU})$ extending from the diaphragm to the right sacroiliac region, with contrast medium concentration. Right retrograde pyelography revealed extensive contrast medium leakage at the upper third of the right ureter. The patient underwent exploratory surgery that revealed communication to the retroperitoneum, dissection of the oblique and transversus muscles, and avulsion of the upper third of the right ureter, with necrotic and inflamed tissue; 7 liters of urine were drained. Subcapsular nephrectomy was performed. The pathology study stated recent multifocal bleeding, sectioned calyces and renal pelvis, the latter with necrotic edges, and inflammatory infiltrate. The patient was released and a tomography scan at 3 postoperative weeks showed a collection at the surgical site that was resolved through percutaneous drainage with no complications or additional incidents.

CONCLUSIONS: Diagnostic delay in cases of urinoma is generally due to the gradual appearance of symptoms and lumbar pain. When infected, they can cause important comorbidity. Standard treatment is often percutaneous drainage, but it can be nephrectomy in some cases.

KEYWORDS: Urinoma; Post-traumatic urinoma
Hospital General Dr. Manuel Gea González (SSA), Ciudad de México.

Correspondence Dr. Ivan Calvo Vázquez dr.ivancv_unam@hotmail.com

\section{ANTECEDENTES}

El urinoma es una colección retroperitoneal de orina, espontánea o secundaria, consucencia de una obstrucción o pérdida de la continuidad de la vía urinaria, con función renal conservada. ${ }^{1}$ La coexistencia de orina fuera del conducto urinario produce lipólisis y estimula una intensa fibrosis, que provoca su encapsulación. Algunos pacientes no manifiestan síntomas, expresan molestias vagas o dolor franco en caso de infección. ${ }^{2,3}$

Con el paso del tiempo se han designado diferentes nombres para esta alteración, por ejemplo: pseudonefrosis (1885, primera descripción), quiste paranéfrico e hidroperinefrosis (1903), hidronefrosis perirrenal (1925), hidrocele renal (1953), pseudohidronefrosis perirrenal (1941), pseudohidronefrosis pararrenal (1948), quiste periureteropélvico y falsa pélvis renal (1953), quiste perinéfrico traumático (1960), pseudoquiste periureteropélvico (1961), quiste perinéfrico (1963), pseudoquiste paraureteral (1963), pseudoquiste pararrenal (1969), hidronefrosis pararrenal (1972), pseudoquiste urinífero perirrenal (1975) y, a partir de 1977, urinoma. $^{4}$

Desde el punto de vista clínico, puede manifestarse con síntomas poco específicos como: dolor abdominal difuso, pérdida de peso o masa palpable; sin embargo, algunos casos suelen ser asintomáticos, incluso expresar complicaciones tardías, como hidronefrosis moderada o severa, íleo paralítico, desequilibrio hidroelectrolítico y formación de un absceso. ${ }^{5}$ 
Cuando la fuga se localiza en el riñón, suele asociarse con alteración o disfunción de los cálices, infundíbulo o pelvis renal, ya sea provocada por algún traumatismo cerrado o penetrante que resulta en lesión renal (desde laceración simple hasta afectación del pedículo vascular) o deberse a obstrucción del sistema genitourinario por litiasis ureteral, masas pélvicas, embarazo, fibrosis retroperitoneal, valvas uretrales y obstrucción vesical. La mayor parte de las lesiones se localizan en la región subcapsular o dentro del espacio perirrenal, incluso pueden extenderse a través del hiato aórtico hacia el mediastino. Cuando se extiende hacia la cavidad intraperitoneal puede rodear las asas intestinales y provocar ascitis urinaria. ${ }^{6}$

Al igual que la fuga renal, la fuga ureteral que origina los urinomas puede deberse a traumatismo cerrado o penetrante, aumento de la presión en el sistema genitourinario por litiasis ureteral, ligadura quirúrgica, masas abdominales o pélvicas; sin embargo, la causa más común es iatrogénica, quizá por cirugía genitourinaria, retroperitoneal, pélvica y ginecológica, o por procedimientos con manipulación endourológica. ${ }^{7}$

Las lesiones vesicales más frecuentes asociadas con los urinomas se originan por algún traumatismo penetrante, con fractura de pelvis, incluso deberse a causas iatrogénicas. Existen dos tipos de lesión vesical: 1) extraperitoneal (65\% de los casos), por alguna laceración vesical por fractura pélvica, o 2) intraperitoneal (35\% de los casos), por traumatismo cerrado. ${ }^{8}$

Los estudios de imagen son importantes para identificar la fuga urinaria, determinar la causa y la extensión del problema. El ultrasonido representa el estudio de primera línea, pero se requiere la complementación diagnóstica con urotomografía. $2,7,9$
El tratamiento de pacientes asintomáticos con urinoma por traumatismo cerrado aún se discute, pues varía desde drenaje percutáneo hasta la colocación del catéter ureteral. ${ }^{10}$ En la mayoría de los casos los urinomas pequeños se reabsorben espontáneamente y no requieren drenaje; sin embargo, en los de gran tamaño, los que persisten durante varios días, y si el paciente manifiesta fiebre o sepsis, independientemente del tamaño, deberá efectuarse el drenaje guiado por imagen. ${ }^{5,6}$

Cuando el urinoma no disminuye de tamaño, aún con la colocación de un catéter de drenaje, puede añadirse un catéter de nefrostomía. Si coexisten lesiones de la vía urinaria, deberá efectuarse la exploración o intervención quirúrgica. ${ }^{5}$

\section{CASO CLÍNICO}

Paciente masculino de 23 años de edad, con antecedente de fractura expuesta de radio y cúbito derecho, provocada por un accidente vehicular, atendido en el servicio de Ortopedia sin complicaciones ni evidencia de lesiones abdominales. Dos meses después del traumatismo acudió al servicio médico por aumento de volumen en la región lumbar y en el flanco derecho de manera progresiva, sin manifestaciones de dolor, fiebre o síntomas urinarios (Figura 1).

La tomografía computada abdominal y pélvica evidenció una colección con densidad líquida (22 UH), amorfa, de bordes lisos y bien delimitados, desde la región perirrenal derecha hasta la sacroiliaca (Figura 2), con desplazamiento cefálico del riñón, que concentró el medio de contraste sin su eliminación (Figura 3). La pielografía ascendente derecha reportó extensa fuga del medio de contraste en el tercio superior del uréter derecho (Figura 4). 


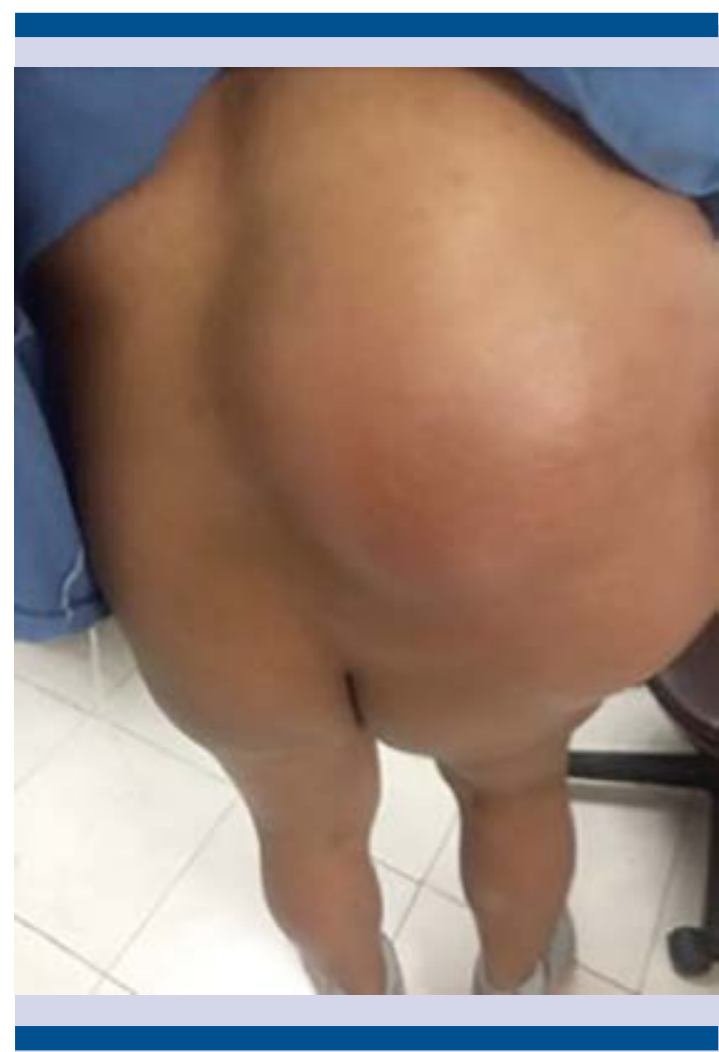

Figura 1. Aspecto clínico del tumor.

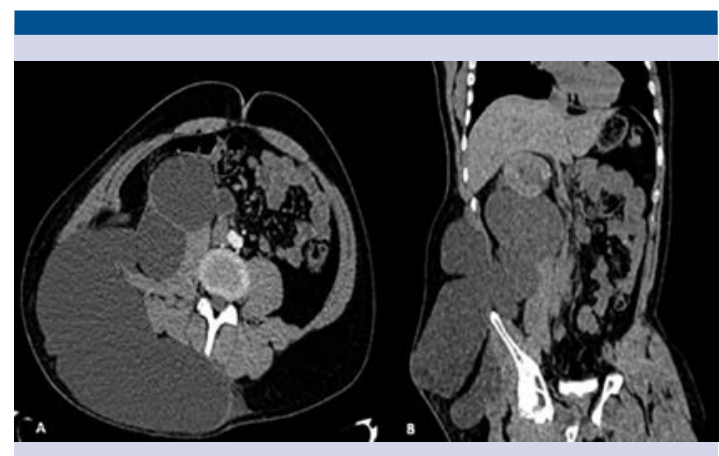

Figura 2. Urotomografía (fase simple): corte transversal (A) y coronal (B).

Se realizó la exploración quirúrgica por lumbotomía. Se drenaron 7 litros de orina, donde se

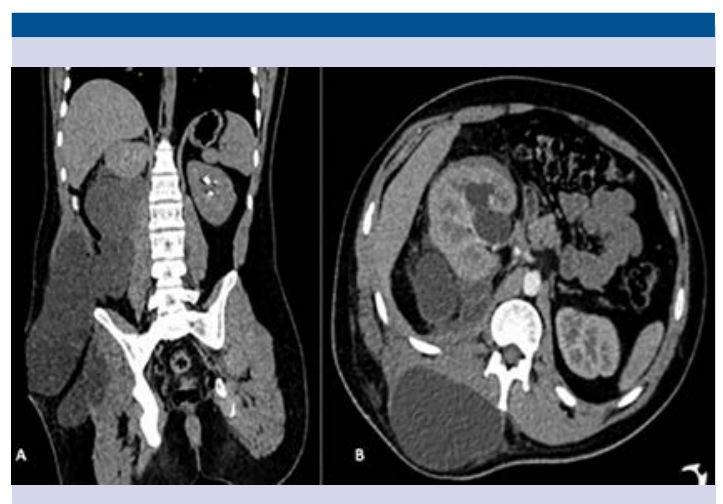

Figura 3. Urotomografía: fase de eliminación (A) y nefrograma (B).

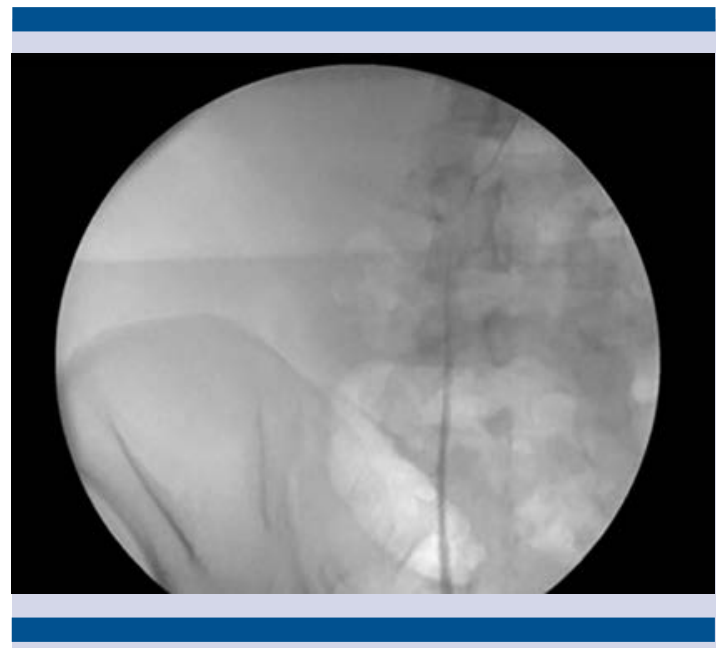

Figura 4. Pielografía ascendente con fuga del medio de contraste en el tercio superior del uréter.

encontró disección del músculo oblicuo y transverso, avulsión del uréter derecho en el tercio superior, con tejido necrótico e inflamatorio. No fue posible localizar el uréter proximal.

El reporte patológico postnefrectomía fue: hemorragia multifocal reciente, cálices y pelvis renal seccionados, bordes necróticos de la pelvis renal e infiltrado inflamatorio, sin encontrar unión ureteropiélica. 
Tres semanas después del posoperatorio, la tomografía computada reportó colección líquida en el sitio quirúrgico, tratada con drenaje percutáneo sin complicaciones ni incidencias adicionales.

\section{DISCUSIÓN}

Las lesiones renales ocurren en $8 \%$ de los pacientes con traumatismo abdominal. El traumatismo cerrado es más común (80-90\%) que el penetrante, principalmente por accidentes vehiculares. La formación de un urinoma es una complicación postraumática excepcional, con incidencia de $1-3 \%$ de los casos. ${ }^{8}$

La formación de un urinoma implica tres condiciones: 1) función renal conservada, 2) ruptura del sistema pielo-calicial o ureteral proximal y 3) obstrucción distal. ${ }^{9,10}$

Ketabchi y sus colaboradores reportaron un caso de urinoma gigante postraumático de un mes de evolución, concomitante con laceración del parénquima renal (grado IV), en el que efectuaron drenaje percutáneo por ultrasonografía, con catéter pigtail $10 \mathrm{~F}$, con drenaje de $3500 \mathrm{~mL}$, con reacción satisfactoria para el paciente. ${ }^{11}$

Los urinomas suelen tratarse con cirugía abierta, pero se recomienda el drenaje percutáneo para prevenir complicaciones serias, como la formación de un absceso y sepsis. ${ }^{12}$

Los pacientes con laceración renal y separación de los fragmentos ureterales requieren exploración quirúrgica, con la finalidad de evitar la formación de un urinoma. ${ }^{13}$

\section{CONCLUSIONES}

La mayor parte de los urinomas se diagnostican tardíamente porque los síntomas aparecen de forma gradual; por tanto, el diagnóstico temprano es clave para el pronóstico del paciente. El diagnóstico de pacientes con urinomas se confirma con estudios de imagen, como ecografía y tomografía computada. El tratamiento de elección consiste en drenaje percutáneo, aunque en pacientes con riñón contralateral sano y ante una gran complejidad al reconstruir el conducto urinario afectado, la nefrectomía es una decisión adecuada.

\section{Financiamiento}

Los autores no recibieron ningún patrocinio para llevar a cabo este estudio.

\section{Conflicto de interés}

Los autores declaran no tener conflicto de interés.

\section{REFERENCIAS}

1. Suárez-Iznaga R, Martí-Moreno MT. Urinoma, un traumatismo olvidado. Rev Cubana Med 2011;50(2):216-221.

2. Dal Mo Yang, et al. Retroperitoneal cystic masses: ct, clinical, and pathologic findings and literature review. Radiographics 2004;24:1353-1365.

3. Puri A, Bajpai M, Gupta AK. Bilateral spontaneous perinephric urinomas: case report and review of the literature. Urology 2004;64:590.E30-590.E32.

4. Mc Inerney D, Jones A, Roylance J. Urinoma. Clin Radiol 1977;28:344-351.

5. Chamalakis K, Prassopoulos P, Hatzidakis A, Gourtsoyiannis $\mathrm{N}$. Chronic intramuscular psoas urinoma presenting with atypical hip symptomatology. [en línea]. Dirección URL: <http://www.eurorad.org/case.php?id=1926>.

6. Titton RL, Gervais DA, Hahn PF, Harisinghani MG, Arellano RS, Mueller PR. Urine leaks and urinomas: diagnosis and imagingguided intervention. Radiographics 2003;23:1133-1147.

7. Christodoulidou M, Clarke L, Napier-Hemy D. Infected urinoma secondary to a ruptured renal calyx from a partial staghorn stone. J Surg Case Rep 2015(8). 10.1093/ jscr/rjv096.

8. Alsikafi NF, McAninch JW, Elliot SP, Garcia M. Nonoperative management outcomes of isolated urinary extravasation following renal lacerations due to external trauma. J Urol 2006;176:2494-2497. 
9. Srinath N, Sood R, Rana K, Madhusoodhanan P. Urinoma following blunt renal trauma. Med J Armed Forces India 2000;56(4):344-346.

10. Philpott J, Nance ML, Carr MC, Canning DA, Stafford PW. Ureteral stenting in the management of urinoma after severe blunt renal trauma in children. J Pediatr Surg 2003;38(7):1096-1098.
11. Ketabchi AA, Ketabchi M, Barkman M. Percutaneous drainage of a late-onset giant posttraumatic urinoma. Urol J 2009;6(3): 214-6.

12. Lee $\mathrm{Y}, \mathrm{Oh} \mathrm{SN}, \mathrm{Rha} \mathrm{SE}$, Byun JY. Renal trauma. Radiol Clin North Am 2007;45(3):581-592.

\section{AVISO PARA LOS AUTORES}

Revista Mexicana de Urología tiene una nueva plataforma de gestión para envío de artículos: https://www.revisionporpares.com/index.php/RMUrol ahí podrá inscribirse a la base de datos administrada por el sistema Open Journal System (OJS) que ofrece las siguientes ventajas para los autores:

- Subir sus artículos directamente al sistema.

- Conocer, en cualquier momento, el estado de los artículos enviados, es decir, si ya fueron asignados a un revisor, aceptados con o sin cambios, o rechazados.

- Participar en el proceso editorial corrigiendo y modificando sus artículos hasta su aceptación final. 